\title{
Mouse lung adenocarcinoma cell lines as tools to identify novel lung cancer genes
}

Nikolaos Kanellakis, Anastasios Giannou, Theodora Agalioti, Rahul Prashanth Ravindran, Dimitra Zazara, loannis Lillis, loanna Giopanou, Malamati Vreka, lan Pavord, Najib Rahman, loannis Psallidas and Georgios Stathopoulos

Background: Carcinogen-induced malignancies, like lung cancer, exhibit high mutation rates. However most mutations remain uncharacterised. (Garraway et al, Cell 2013;153:17-37)

Aim: To establish a reliable mouse model of cigarette carcinogen induced human lung adenocarcinoma appropriate for the discovery of novel lung cancer genes, mutations and pathways.

Methods: We developed a strategy to obtain mouse lung adenocarcinoma cell lines (MLA) by repeatedly exposing FVB, C57BL/6 and Balb/c mouse strains to the tobacco carcinogens urethane or diethylnitrosamine. All MLA were subjected to global gene expression profiling, and screened for the expression of known oncogenes and cancer stemness markers.

Results: The resulting MLA cell lines were phenotypically steady over the passages, immortal and displayed sustained cellular proliferation in minimally-supplemented conditions. Moreover, all MLA cells appeared to be transplantable, oncogenic, metastatic, lethal in vivo and able to form tumorspheres, a capacity unique to tumor and stem cells. Cancer stemness factors Itgb3 and Lgr6 revealed high expression levels. Remarkably MLA cells exhibited major characteristics of the human lung cancer, such Kras mutations in codon 61, and mono- or bi-allelic loss of the tumor suppressor Trp53. A pilot microarray and RNA-Sequencing study identified focal overexpression of Prl2c2, encoding proliferin (PRL), selectively by lung adenocarcinoma cells. To this end Prl2c2 knock-down MLA cells displayed reduced proliferation rate.

Conclusions: MLA cells are promising models of human lung adenocarcinoma. Our studies identified Prl2c2 as a candidate lung tumor promoter.

Funding: European Research Council \#260524, European Respiratory Society \#STRTF 2015 - 9508 